\title{
The Prognostic Value of Systemic Inflammation Response Index in Cholangiocarcinoma Patients
}

\author{
Bao Jin ${ }^{1} * *$ \\ Wenmo $\mathrm{Hu}^{2}$,* \\ $\mathrm{Si} \mathrm{Su}{ }^{2, *}$ \\ Haifeng $X u^{\prime}$ \\ Xin Lu' \\ Xinting Sang $\mathbb{D}^{\prime}$ \\ Huayu Yang $\mathbb{D}^{\prime}$ \\ Yilei Mao (D) \\ Shunda Du (1D) \\ 'Department of Liver Surgery, Peking \\ Union Medical College Hospital \\ (PUMCH), Peking Union Medical College \\ (PUMC) \& Chinese Academy of Medical \\ Sciences (CAMS), Beijing, I00730, \\ People's Republic of China; ${ }^{2}$ Peking Union \\ Medical College (PUMC) \& Chinese \\ Academy of Medical Sciences (CAMS), \\ Beijing, 100730, People's Republic of \\ China
}

*These authors contributed equally to this work
Correspondence: Shunda Du; Yilei Mao Department of Liver Surgery, Peking Union Medical College (PUMC) Hospital, PUMC \& Chinese Academy of Medical Sciences, Beijing, 100730, People's

Republic of China

Email dushd@pumch.cn;

pumch-liver@hotmail.com
Purpose: We determined the prognostic value of the systemic inflammation response index (SIRI) in patients with cholangiocarcinoma after surgery and constructed a survival prediction model based on SIRI.

Patients and Methods: We recruited 328 patients with histopathologically confirmed cholangiocarcinoma from 2003 to 2017 and performed Kaplan-Meier survival and Cox analyses to analyze the prognostic value of the SIRI and identify other significant factors. A nomogram involving SIRI and other clinicopathological factors was established based on the training cohort. The concordance index (C-index), decision curve analysis, calibration plots, and HosmerLemeshow test were used to evaluate the clinical utility of the nomogram and to compare it with the traditional TNM staging system. The results were validated using a separate validation cohort. Results: The patients were randomly divided into the training $(\mathrm{n}=232)$ and validation $(\mathrm{n}=$ 96) cohorts. In the training cohort, the independent factors derived from the Cox multivariate analysis were SIRI, platelet-to-lymphocyte ratio, jaundice, $\gamma$-glutamyl transpeptidase level, maximal tumor size, $\mathrm{N}$ stage, $\mathrm{M}$ stage, and radical surgery. Time-dependent receiver operating characteristic (ROC) curves showed higher AUC for SIRI than those for other inflammation-based biomarkers. A nomogram containing all the independent factors showed good discrimination and calibration. The C-index values for overall survival, $0.737(95 \% \mathrm{Cl}$ : $0.683-0.791)$ and $0.738(95 \% \mathrm{Cl}: 0.679-0.797)$ in the training and validation cohorts, respectively, were significantly better than those for the TNM staging system $[0.576$ (95\% $\mathrm{Cl}: 0.515-0.637)$ and 0.523 (95\% Cl: 0.465-0.581), respectively].

Conclusion: SIRI was an independent prognostic factor for cholangiocarcinoma. A prognostic model based on SIRI might help clinicians to stratify patients more precisely and provide individualized treatment.

Keywords: systemic inflammation response index, cholangiocarcinoma, prognosis, nomogram, survival

\section{Introduction}

Cholangiocarcinoma, the second most common hepatobiliary malignant tumor after hepatocellular carcinoma, ${ }^{1}$ originates from the epithelium of the bile ducts and can involve any part of the biliary tree. ${ }^{2}$ The incidence of cholangiocarcinoma has reportedly been increasing in recent years, ${ }^{3,4}$ with age-adjusted rates between 2.1 per 100,000 in black and non-Hispanic populations and 2.8-3.3 per 100,000 in Asian and Hispanic people. ${ }^{1}$ Since most patients at the early stage are asymptomatic, a large proportion have advanced-stage disease at presentation. Therefore, only approximately $35 \%$ of patients at an early stage have the chance to undergo surgical resection with curative intent. ${ }^{5}$ Moreover, nearly $30 \%$ of patients judged to be resectable on initial imaging tests are unresectable during exploration, resulting in positive margins in the final 
pathology reports. ${ }^{6}$ All these factors contribute to the poor prognosis of cholangiocarcinoma patients, and the mortality is parallel to its incidence rate (approximately 1.0 per 100,000). ${ }^{7,8}$ Thus, precise prediction of the outcome of patients with cholangiocarcinoma is urgently needed. The TNM staging system is currently the most widely used standard for cancer staging. Nevertheless, other clinicopathological factors might also play crucial roles in the outcome of patients with cholangiocarcinoma, leading to considerable heterogeneity in clinical prognosis among patients with the same stage. Hence, more accurate prognostic models for cholangiocarcinoma are urgently needed in the clinical setting.

It is widely recognized that the inflammatory response is an important factor in cancer development and progression. ${ }^{9}$ Information about some immune-inflammatory cells, such as neutrophils, monocytes, platelets, and lymphocytes, can be easily acquired by performing a complete blood count test. Based on this, inflammation-based biomarkers including neutrophil-to-lymphocyte ratio (NLR), monocyte-to-lymphocyte ratio (MLR), and platelet-to-lymphocyte ratio (PLR) have been investigated, and their prognostic value in cholangiocarcinoma has been illustrated. ${ }^{10,11}$ Recently, Qi et al proposed an innovative inflammation-based biomarker integrating peripheral counts of neutrophils, monocytes, and lymphocytes, namely the systemic inflammation response index (SIRI). ${ }^{12}$ Subsequent studies have reported the prognostic ability of SIRI in solid tumors, such as esophageal, gastric, pancreatic, and nasopharyngeal cancers. ${ }^{13-15}$ However, there is still a lack of evidence showing whether SIRI can be a prognostic indicator for predicting the outcome of patients with cholangiocarcinoma. Additionally, to the best of our knowledge, there is still no prognostic model that includes inflammation-based biomarkers for cholangiocarcinoma.

Therefore, our research aimed to study the prognostic value of SIRI using a cohort of cholangiocarcinoma patients after surgical resection, and to construct a prognostic model integrating the SIRI in a training cohort and test its predictive accuracy in a validation cohort.

\section{Patients and Methods}

\section{Patients}

From January 1, 2003, to December 31, 2017, patients who received surgical treatment at our institution and whose pathological report revealed cholangiocarcinoma were recruited retrospectively for this study.
The study's inclusion criteria were: (1) cholangiocarcinoma as the primary diagnosis, which was confirmed through histopathological examination, (2) surgery performed for cholangiocarcinoma, (3) the results of routine blood tests measured within seven days before surgery were available, and (4) the postoperative follow-up data and clinicopathological information were complete and available.

The study's exclusion criteria were: (1) lack of definitive histopathological diagnosis of cholangiocarcinoma, (2) missing clinicopathological information, (3) incomplete follow-up data, and (4) the comorbid presence of other malignant tumors.

After screening, the 328 patients who were subsequently enrolled met the inclusion criteria. The entire cohort was randomly divided into training and validation cohorts in a 7:3 ratio using the "caret" package of $\mathrm{R}$ version 3.6.2.

\section{Ethics Statement}

This study was approved by the Institutional Review Board of the Peking Union Medical College Hospital (PUMCH) (No: S-K1110). Informed consent was obtained from all the patients according to the Committee's regulations. The study was performed in accordance with the ethical standards of the Declaration of Helsinki. ${ }^{16}$

\section{Data Collection}

Related medical records were manually reviewed to obtain demographic and clinical information. We collected the patients' demographic information, clinical symptoms, serum test results, surgical reports, and histopathological results. Inflammatory biomarkers, including the SIRI, NLR, MLR, and PLR, were defined as follows: SIRI = $\mathrm{N}^{*} \mathrm{M} / \mathrm{L}, \mathrm{NLR}=\mathrm{N} / \mathrm{L}, \mathrm{MLR}=\mathrm{M} / \mathrm{L}$, and $\mathrm{PLR}=\mathrm{P} / \mathrm{L}$, where $\mathrm{N}$, $\mathrm{M}, \mathrm{L}$, and $\mathrm{P}$ refer to the peripheral counts of neutrophils, monocytes, lymphocytes, and platelets, respectively. The 7th edition of the AJCC TNM classification system was used to determine the preoperative clinical and postoperative pathological TNM stages. ${ }^{17}$ The incisional margins and maximal tumor size were determined based on the final histopathological reports. Radical surgery was defined as the requirement of radical surgical protocols with microscopically negative incisional margins. All patients underwent regular follow-ups after discharge. The time of the last follow-up was June 2020, and the survival status of the patients was recorded. The overall 
survival (OS) was calculated based on the time interval from the time of surgery to the time of death or the last follow-up.

\section{Statistical Analysis}

Continuous variables are presented as median, first, and third quartiles, while categorical variables are expressed as numbers and percentages. According to the routine cutoff value in clinical applications, continuous variables such as carbohydrate antigen 19-9 (CA19-9) and carcinoembryonic antigen (CEA) were converted into categorical variables. The optimal cutoff value for inflammation-based biomarkers for OS was calculated using x-tile software. ${ }^{18}$ The Kaplan-Meier method was used to draw the survival curves. Differences between subgroups were compared using Log rank tests. The patients were divided into high- and low-SIRI groups according to the optimal SIRI cutoff value. The correlations between clinicopathological variables and the SIRI groups were analyzed using twosample t- or Mann-Whitney $U$-tests for continuous variables based on normality and Pearson chi-squared or Fisher's exact tests for categorical variables. All statistical analyses were performed using $\mathrm{R}$ software (version 4.0.2, Institute of Statistics and Mathematics, Vienna, Austria) and SPSS software (version 25.0, IBM Corp., Armonk, NY, USA). A two-sided $P$ value of $<0.05$, was considered statistically significant.

In the training cohort, the Cox regression method was applied to the univariate analysis. The multivariate Cox proportional hazards regression model was then applied to the variables with $\mathrm{P}$ values not greater than 0.1 , in univariate analysis. The covariates included in the prediction model were selected based on the results of Cox regression analysis. A prognostic nomogram was established to provide a predictive model for use as a calculation tool for predicting OS.

In the validation cohort, the prediction model was verified by measuring the discrimination ability and calibration. We used Harrell's concordance index (C-index) to measure the discrimination ability. The validation was based on 1000 bootstrap resamplings. The calibration of the model was assessed using calibration plots, and the calibration effect was evaluated with the HosmerLemeshow test. The C-index was also used to compare the discrimination abilities of the prediction model and the traditional TNM staging system. A decision curve analysis (DCA) was also carried out to determine the clinical usefulness by calculating the net benefits at different threshold probabilities. ${ }^{19}$

\section{Results}

\section{Baseline Characteristics of Patients}

This study investigated 328 patients with cholangiocarcinoma. In 224 (68.3\%) patients, the tumor was located in the extrahepatic site, and the rest had intrahepatic cholangiocarcinoma. Baseline clinicopathological characteristics of the study patients for the whole $(n=328)$, training $(n=232)$, and validation $(n=96)$ cohorts are shown in Table 1. In the entire cohort, 196 (59.8\%) patients were men and $132(40.2 \%)$ were women. One-hundredseventeen $(35.7 \%)$ patients were aged 65 years or older when they underwent surgery. Forty-six (14.0\%), 78 (54.3\%), and 231 (70.4\%) patients developed fever, weight loss, and jaundice, respectively, as diagnosed with cholangiocarcinoma. Elevated serum CA19-9 and CEA levels were observed in $254(77.4 \%)$ and 60 (18.3\%) patients, respectively. According to the AJCC 7th edition, one (0.3\%), 129 (39.3\%), 119 (36.3\%), 54 (16.5\%), and 25 (7.6\%) patients had stage 0, I, II, III, and IV disease, respectively. Surgical resection with curative intent was achieved in $183(55.8 \%)$ patients; the remaining patients did not undergo radical surgery. For 25 (7.6\%) patients with TNM stage IV disease, palliative surgery was performed to relieve patient symptoms. The median follow-up time was 18.5 months (range form 1.0-192.0 months). Finally, 213 patients (64.9\%) died.

\section{Clinicopathological Characteristics According to SIRI}

The optimal cutoff values of SIRI, MLR, NLR, and PLR generated using the x-tile software were 0.68, 0.24, 3.15 , and 106.20 , respectively. In the training and validation cohorts, the patients were divided into high SIRI $(>0.68)$ and low SIRI $(\leq 0.68)$ groups based on the optimal SIRI cutoff value. Table 1 summarizes the characteristics of each patient. Compared to patients in the low SIRI group in both the training and validation cohorts, the high SIRI group had more patients aged $\geq$ 65 years and more patients with higher MLR, higher NLR, and elevated serum lactate dehydrogenase (LDH) levels. In the training cohort, the mortality rate increased from $42.9 \%$ to $72.7 \%$ between the low and high SIRI groups $(\mathrm{p}=0.000)$, while that in the validation cohort increased from $43.5 \%$ to $69.6 \%(p=0.022)$. In the 
Table I Baseline Characteristics of All Patients

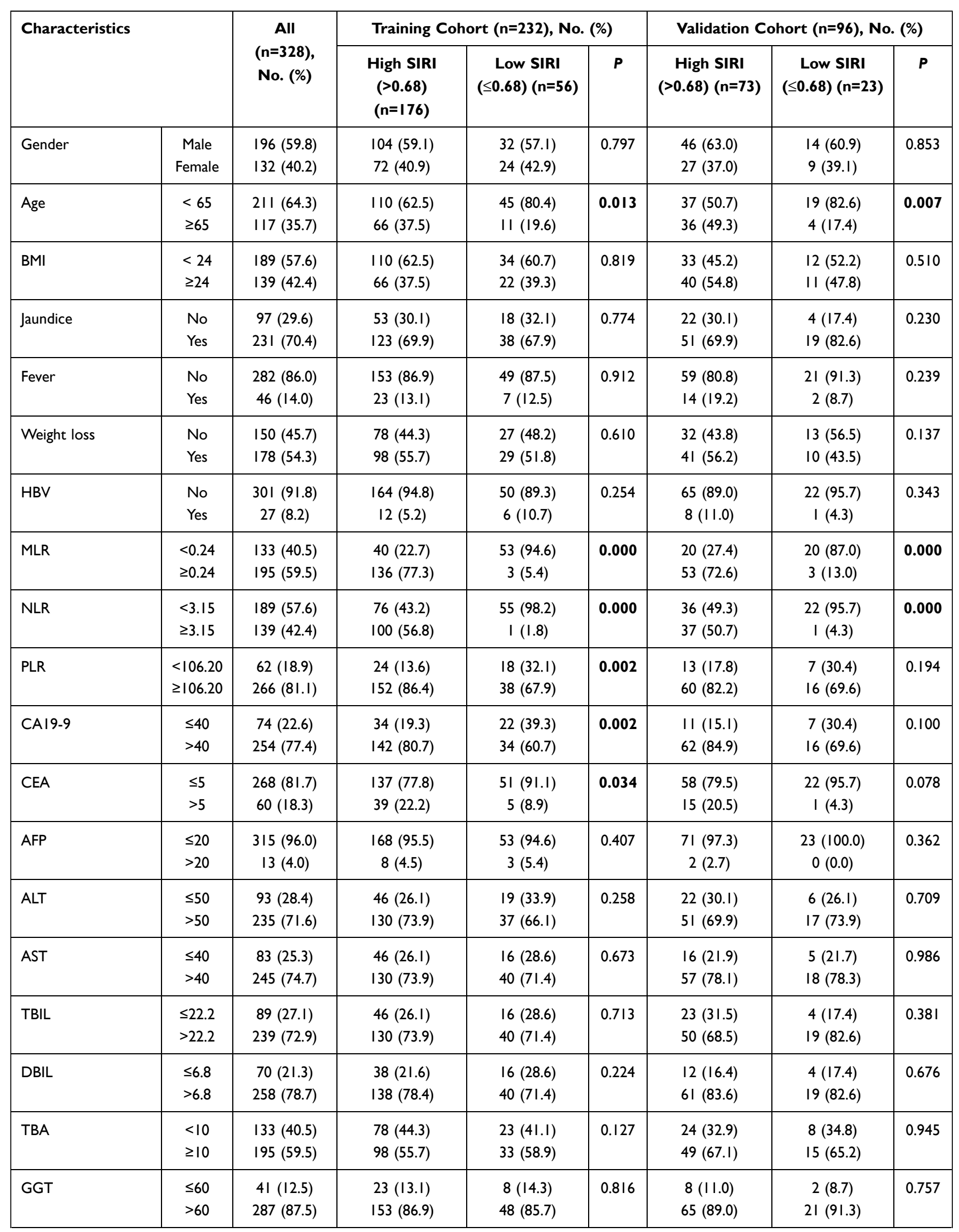

(Continued) 
Table I (Continued).

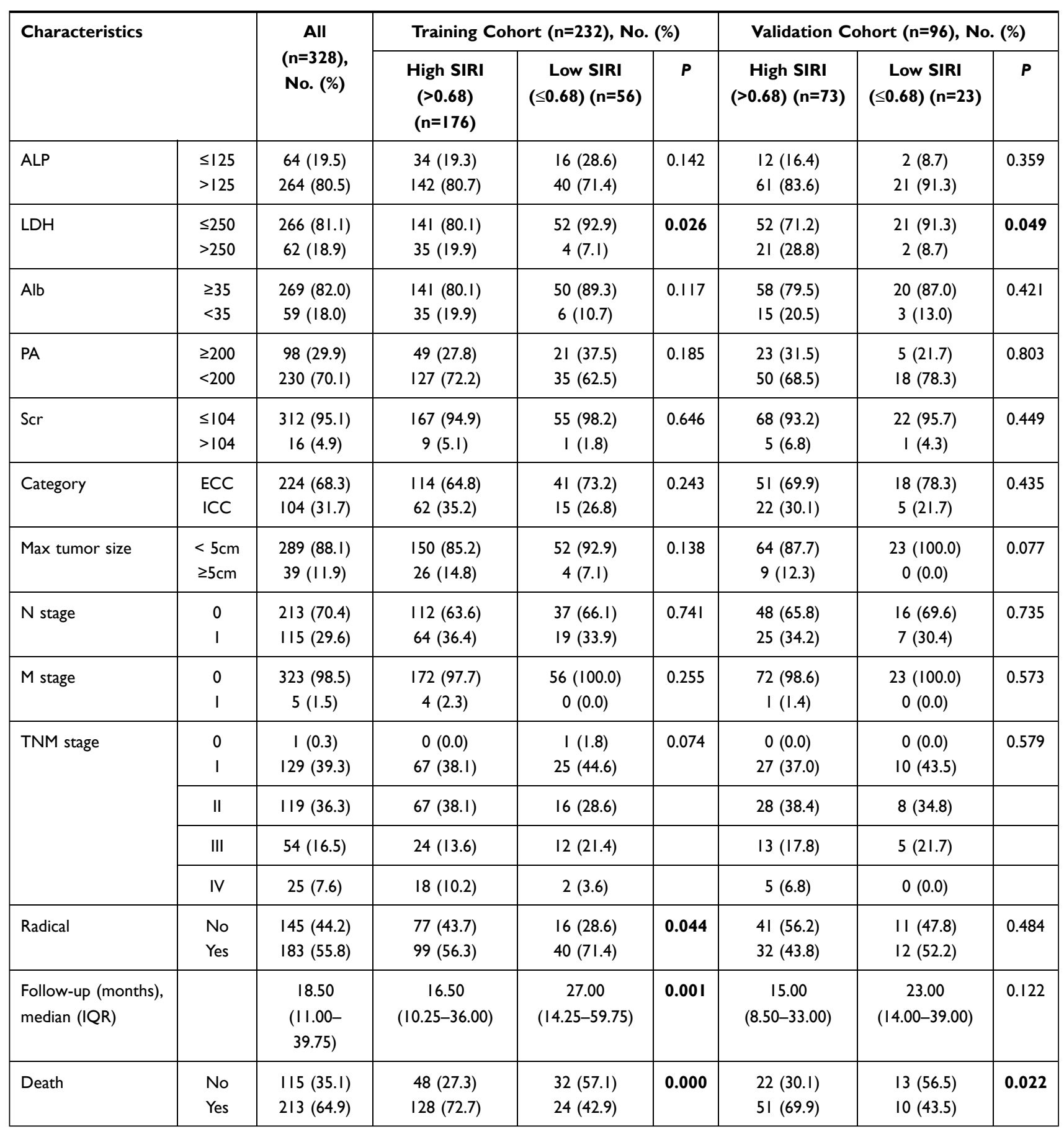

Note: The bold text indicates that the $P$ value is less than 0.05 .

Abbreviations: SIRI, systemic inflammation response index; CA19-9, carbohydrate antigen 19-9; CEA, carcinoembryonic antigen; AFP, alpha-fetoprotein; TNM, tumornode-metastasis; BMI, body mass index; HBV, hepatitis B virus; MLR, monocyte-to-lymphocyte ratio; NLR, neutrophil-to-lymphocyte ratio; PLR, platelet-to-lymphocyte ratio; ALT, alanine aminotransferase; AST, aspartate aminotransferase; TBIL, total bilirubin; DBIL, direct bilirubin; TBA, total bile acids; GGT, $\gamma$-glutamyl transpeptidase; ALP, alkaline phosphatase; LDH, lactate dehydrogenase; Alb, albumin; PA, prealbumin; Scr, serum creatinine; ECC, extrahepatic cholangiocarcinoma; ICC, intrahepatic cholangiocarcinoma.

training cohort, more patients in the high SIRI group experienced elevated CA19-9 $(\mathrm{p}=0.002)$ and CEA $(p=0.034)$ levels; fewer patients underwent radical surgery $(56.3 \%$ vs $71.4 \%, \mathrm{p}=0.044)$ and a decreased median follow-up time was observed (16.50 vs 27.00 months, $\mathrm{p}=0.001$ ) compared to those in the low SIRI group; no significant difference was found in the validation cohort. 


\section{The Predictive Factors of OS}

In the training cohort, the OS Kaplan-Meier curves based on the SIRI showed significant differences, which was confirmed by the Log rank test $(P=0.00$; Figure $1 \mathrm{~A})$. The same result was observed in the validation cohort $(P=0.03$; Figure 1B). Moreover, we divided the entire cohort based on tumor location (extrahepatic cholangiocarcinoma [ECC] or intrahepatic cholangiocarcinoma [ICC]) and surgery type (radical or palliative). The OS Kaplan-Meier curves according to the SIRI in the above-mentioned subcohorts also showed significant differences (Figure 1C-F). We then performed Cox proportional hazards analysis for OS in the training cohort (Table 2). In univariate analysis, jaundice, CA19-9, $\gamma$-glutamyl transpeptidase (GGT), alkaline phosphatase (ALP), LDH, albumin (Alb), maximal tumor size, $\mathrm{N}$ stage, $\mathrm{M}$ stage, radical surgery, and all the inflammation-based biomarkers were statistically associated with OS $(\mathrm{p}<0.1)$. Then the multivariate analysis showed that jaundice (hazard ratio [HR]:2.0980; 95\% confidence interval [CI]: 1.1567-3.805; $P=0.015$ ), high SIRI (HR:1.8892; 95\% CI: 1.0870-3.2836; $P=0.002$ ), PLR (HR:1.9085; 95\% CI: 1.1392-3.1972; $P=0.017)$, GGT (HR:2.38; 95\% CI: 1.1313-5.0072; $P=0.022$ ), maximal tumor size (HR:2.5509; 95\% CI: 1.3096-4.969; $P=0.006$ ), N stage (HR:1.8034; 95\% CI: 1.2337-2.6364; $P=0.002$ ), M stage (HR:4.3937; 95\% CI: $1.1853-16.2874$; $P=0.027$ ), and radical surgery (HR:0.3675; 95\% CI: $0.2558-0.528 ; P=0.000$ ) were independent factors to predict OS.

\section{The Establishment of Prediction Model for OS}

The prognostic model for OS was established using variables obtained from the multivariate analysis. The prognostic clinicopathological factors included eight risk factors, including SIRI, PLR, jaundice, GGT, maximal tumor size, $\mathrm{N}$ stage, $\mathrm{M}$ stage, and radical surgery. The time-dependent receiver operating characteristic (ROC) curves of the prognostic model including all eight risk factors, the prognostic model excluding SIRI and PLR, and the TNM staging system are shown in Figure 2A, which shows that the model integrating all eight factors was much more reliable in predicting OS than the TNM staging system, with 1-, 3-, and 5-year areas under the ROC curves (AUCs) of $0.799,0.802$, and 0.867 , respectively (AUCs of TNM staging system in 1-, 3-, and 5-years: $0.607,0.593$, and 0.602 , respectively). The model integrating all factors also had larger AUCs within the full-time range than those for the model without SIRI and PLR. Furthermore, the AUCs of SIRI were also higher than those of the other inflammation-based biomarkers, including MLR, NLR, and PLR (Figure 2B). We then constructed a nomogram containing all these eight factors to make the prognostic model more visualized and practical, as shown in Figure 3. We assessed the predictive ability of the model in the training cohort using the $\mathrm{C}$-index, which was $0.737(95 \% \mathrm{Cl}$ : 0.683-0.791), indicating good discrimination accuracy of the nomogram. The C-index of the TNM staging system in the training cohort was $0.576(95 \% \mathrm{Cl}$ : 0.515-0.637). Furthermore, the performance of the nomogram was evaluated using 1-, 3-, and 5-year calibration plots (Figure 4A, C and E). The predicted and reference lines had a high degree of coincidence, indicating good performance of the model (HosmerLemeshow test, $P=0.458,0.813$ and 0.205 for $1-, 3-$, and 5-year OS in the training cohort, respectively).

\section{Validation of the Predictive Accuracy of the Nomogram}

In the validation cohort, the AUC of the nomogram for 1, 3 , and 5 years was $0.822,0.784$, and 0.842 , respectively (the corresponding AUC of the TNM staging system was $0.566,0.582$, and 0.590 , respectively). Moreover, the C-index of the nomogram for OS prediction $(0.738,95 \%$ Cl: 0.679-0.797) was significantly higher than that of the TNM staging system $(0.523,95 \% \mathrm{Cl}: 0.465-0.581$, $P<0.05)$. The 1-, 3-, and 5-year calibration plots (Figure $4 \mathrm{~B}, \mathrm{D}$ and $\mathrm{F}$ ) for the probability of OS also showed an optimal agreement between actual observation and prediction using the prognostic model (HosmerLemeshow test, $P=0.322,0.174$ and 0.792 for 1-, 3-, and 5-year OS in the validation cohort, respectively). In addition, DCA for the prognostic model and TNM staging system (Figure 5) showed that our prognostic model was more beneficial than all or none of the patients' dead scheme if the threshold probability of a patient was above $30 \%$. Moreover, in this range, our model provided more benefits than the TNM staging system. In addition, compared to the model without SIRI and PLR, the prognostic model integrating all the factors also had preferable clinical utility, except for the 5-year DCA in the validation cohort. 
A

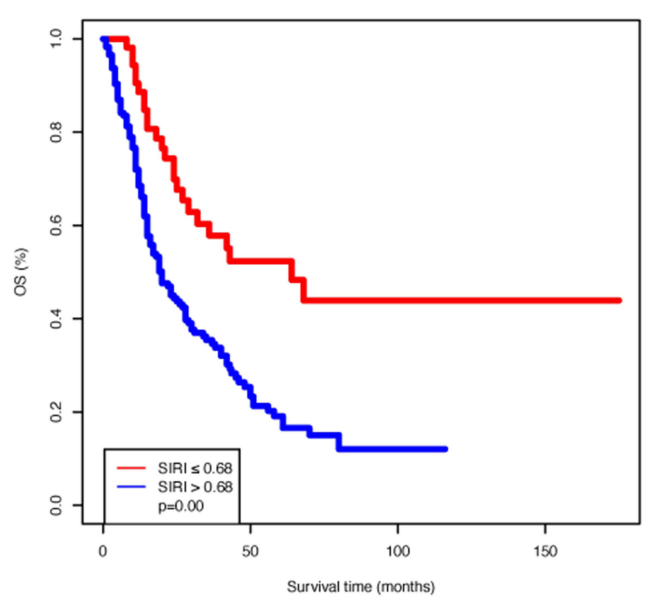

C

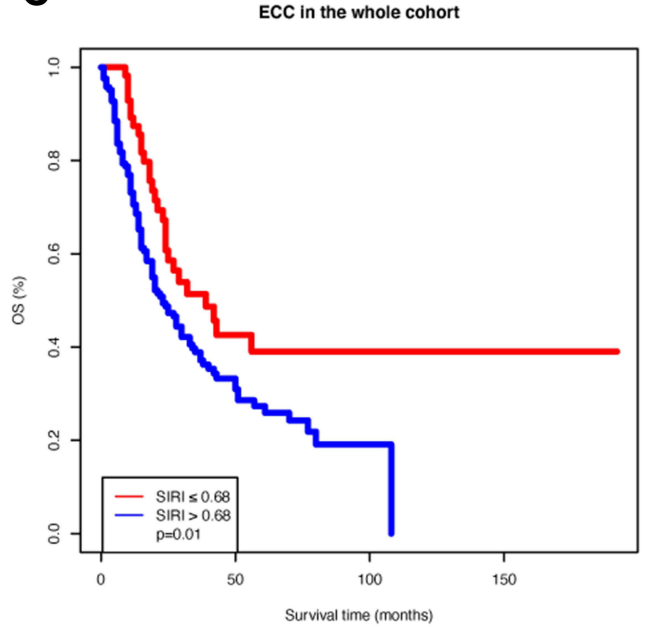

E

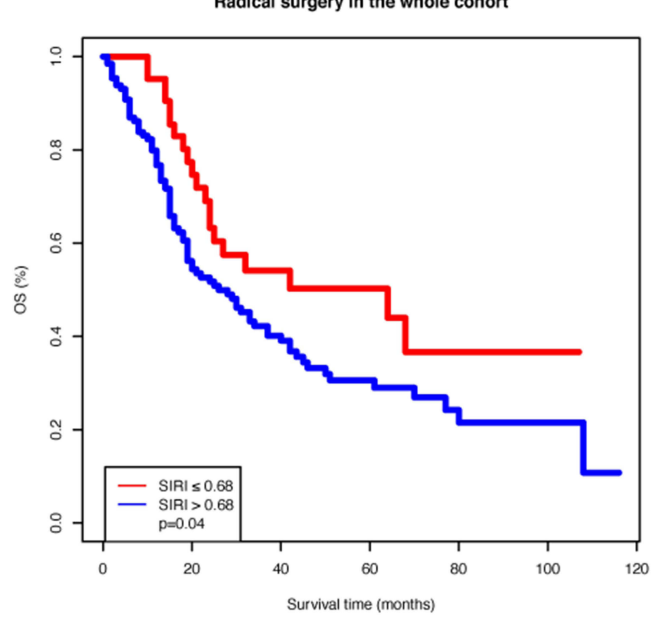

B

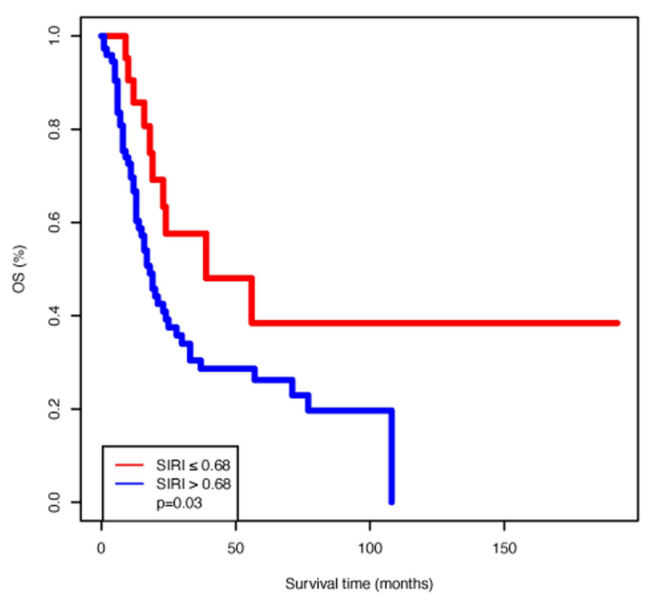

D

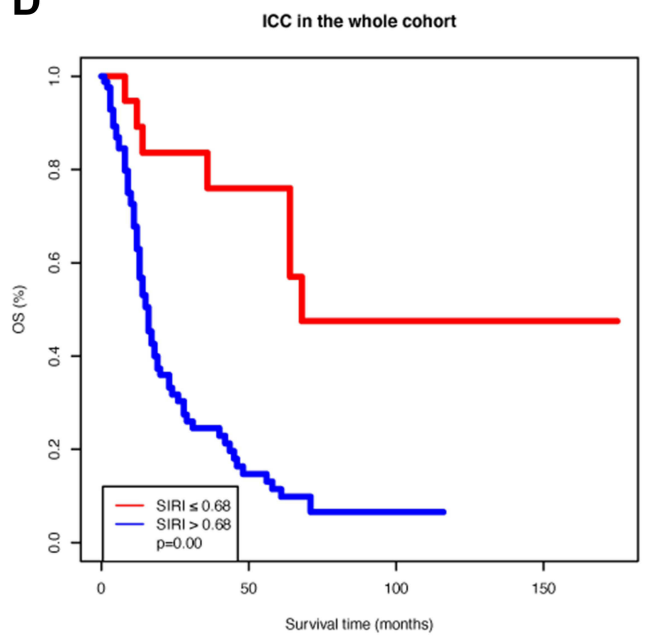

$\mathbf{F}$

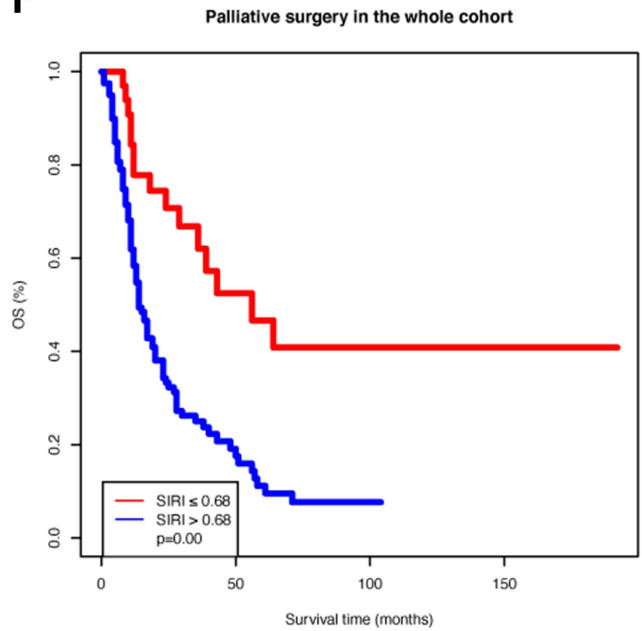

Figure I Kaplan-Meier survival curves of different systemic inflammation response index (SIRI) groups. (A) Training cohort; (B) Validation cohort; (C) Patients with extrahepatic cholangiocarcinoma; (D) Patients with intrahepatic cholangiocarcinoma; (E) Patients who received radical surgery; (F) Patients who underwent palliative surgery. 
Table 2 Univariate and Multivariate Cox Proportional Hazards Analysis for OS in the Training Cohort

\begin{tabular}{|c|c|c|c|c|c|c|c|}
\hline \multicolumn{2}{|l|}{ Characteristics } & \multicolumn{3}{|c|}{ Univariate Analysis } & \multicolumn{3}{|c|}{ Multivariate Analysis } \\
\hline & & \multirow{4}{*}{$\begin{array}{c}\text { HR } \\
1.003 \\
1.158 \\
0.9426\end{array}$} & \multirow{4}{*}{$\begin{array}{c}95 \% \mathrm{Cl} \\
0.7242-1.389 \\
0.8304-1.615 \\
0.6545-1.358\end{array}$} & \multirow{4}{*}{$\begin{array}{c}P \\
0.987 \\
0.387 \\
0.751\end{array}$} & \multirow[t]{4}{*}{ HR } & \multirow[t]{4}{*}{$95 \% \mathrm{Cl}$} & \multirow[t]{4}{*}{$\mathbf{P}$} \\
\hline Demographics & Sex (female/male) & & & & & & \\
\hline & Age $(\geq 65 /<65)$ & & & & & & \\
\hline & BMI $(\geq 24 /<24)$ & & & & & & \\
\hline \multirow[t]{3}{*}{ Symptoms } & Jaundice (YES/NO) & 1.537 & $1.067-2.215$ & 0.021 & 2.0980 & $1.1567-3.805$ & 0.015 \\
\hline & Weight loss (YES/NO) & 1.151 & $0.835-1.587$ & 0.39 & & & \\
\hline & Fever (YES/NO) & 0.9985 & $0.6094-1.636$ & 0.995 & & & \\
\hline Past medical history & HBV (YES/NO) & 1.323 & $0.7466-2.343$ & 0.338 & & & \\
\hline \multirow[t]{5}{*}{ Inflammation-based biomarkers } & SIRI (high/low) & 2.42 & $1.56 \mid-3.753$ & 0.000 & 1.8892 & $1.0870-3.2836$ & 0.002 \\
\hline & MLR $(\geq 0.24 /<0.24)$ & 1.654 & $1 .|84-2.3|$ & 0.00317 & 0.9615 & $0.6280-1.4720$ & 0.866 \\
\hline & $\operatorname{NLR}(\geq 3.15 /<3.15)$ & 1.764 & $1.28-2.43$ & 0.000523 & 1.3753 & $0.9014-2.0984$ & 0.139 \\
\hline & PLR $(\geq 106.20 /<106.20)$ & 2.065 & I.276-3.344 & 0.00317 & 1.9085 & $1.1392-3.1972$ & 0.017 \\
\hline & $\mathrm{PNI}(\geq 44.40 /<44.40)$ & 0.6301 & $0.4546-0.8734$ & 0.00556 & 1.3382 & $0.8510-2.1044$ & 0.207 \\
\hline \multirow[t]{14}{*}{ Blood test } & CAI99(>40/ $\leq 40)$ & 1.85 & $1.217-2.81$ & 0.00396 & 1.2028 & $0.6924-2.0892$ & 0.512 \\
\hline & CEA $(>5 / \leq 5)$ & 1.814 & $1.24-2.654$ & 0.00216 & & & \\
\hline & $\operatorname{AFP}(>20 / \leq 20)$ & 1.91 & $0.884-4.125$ & 0.0997 & & & \\
\hline & $\operatorname{ALT}(>50 / \leq 50)$ & 1.112 & $0.7765-1.592$ & 0.563 & & & \\
\hline & AST $(>40 / \leq 40)$ & 0.9686 & $0.6762-1.387$ & 0.862 & & & \\
\hline & TBIL $(>22.2 / \leq 22.2)$ & 1.273 & $0.8677-1.867$ & 0.217 & & & \\
\hline & DBIL $(>6.8 / \leq 6.8)$ & 1.409 & $0.9398-2.113$ & 0.0969 & & & \\
\hline & TBA $(\geq 10 /<10)$ & 1.279 & $0.9105-1.796$ & 0.156 & & & \\
\hline & GGT $(>60 / \leq 60)$ & 1.793 & $1.067-3.015$ & 0.0275 & 2.38 & $1.1313-5.0072$ & 0.022 \\
\hline & $\operatorname{ALP}(>125 / \leq 125)$ & 1.379 & $0.919-2.07$ & 0.121 & 0.5520 & $0.278 \mathrm{I}-1.0957$ & 0.089 \\
\hline & $\mathrm{LDH}(>250 / \leq 250)$ & 1.431 & $0.9482-2.161$ & 0.0879 & 1.1490 & $0.727|-1.8| 58$ & 0.552 \\
\hline & Alb $(\geq 35 /<35)$ & 0.5364 & $0.360 \mathrm{I}-0.799$ & 0.00218 & 0.6561 & $0.3858-1.1159$ & 0.120 \\
\hline & $\mathrm{PA}(\geq 200 /<200)$ & 0.9808 & $0.6826-1.409$ & 0.916 & & & \\
\hline & Scr $(>104 / \leq 104)$ & 1.209 & $0.6585-2.219$ & 0.541 & & & \\
\hline \multirow[t]{4}{*}{ Post-operation } & Tumor size $(>5 / \leq 5)$ & 1.503 & $0.9637-2.344$ & 0.0723 & 2.5509 & $1.3096-4.969$ & 0.006 \\
\hline & $\mathrm{N}$ stage $(\mathrm{I} / 0)$ & 2.028 & $1.46 \mathrm{I}-2.815$ & 0.000 & 1.8034 & $1.2337-2.6364$ & 0.002 \\
\hline & M stage $(\mathrm{I} / 0)$ & $|5.9|$ & $5.535-45.75$ & 0.000 & 4.3937 & I.1853-16.2874 & 0.027 \\
\hline & Radical (Yes/No) & 0.385 & $0.2786-0.5319$ & 0.000 & 0.3675 & $0.2558-0.528$ & 0.000 \\
\hline
\end{tabular}

Note: The bold text indicates that the $P$ value is less than 0.05 .

\section{Comparison of the Predictive Accuracy for OS Between the Nomogram and the TNM Staging System}

Based on the prognostic model developed in this study, we divided the entire cohort into four groups with different risks. The OS Kaplan-Meier curves according to the prognostic model's risk demonstrated significant differences, which was confirmed by the Log rank test $(P=0.00$, Figure 6A). In addition, the prognostic classification of the model was even better than that of the TNM staging system (Figure 6B), with more separated curves and no crossing lines in the full-time range. Moreover, in patients with ECC or ICC, our model also showed better prognostic prediction performance than that of the TNM staging system (Figure 6C-F).

\section{Discussion}

The incidence of cholangiocarcinoma has been reported to increase in recent years. $^{20,21}$ Although radical surgery remains the only curative option, many patients are diagnosed at an advanced stage, thus losing the opportunity for a cure. Multiple factors contribute to the current poor prognosis, with very low 5-year survival rates and cumulative mortality rates increasing by $39 \% .^{22,23}$ Thus, an accurate tool for predicting the prognosis of patients with 
A

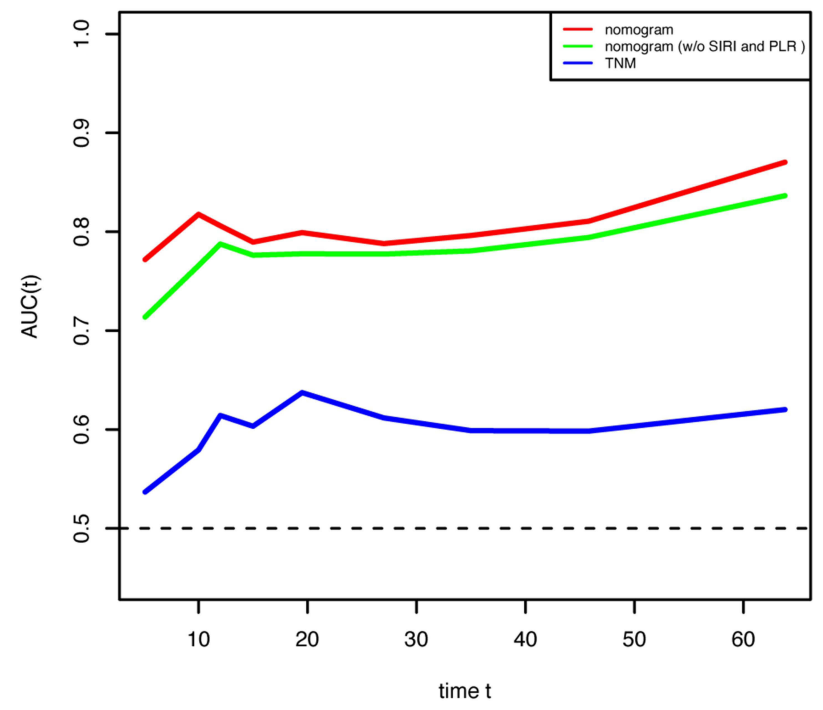

B

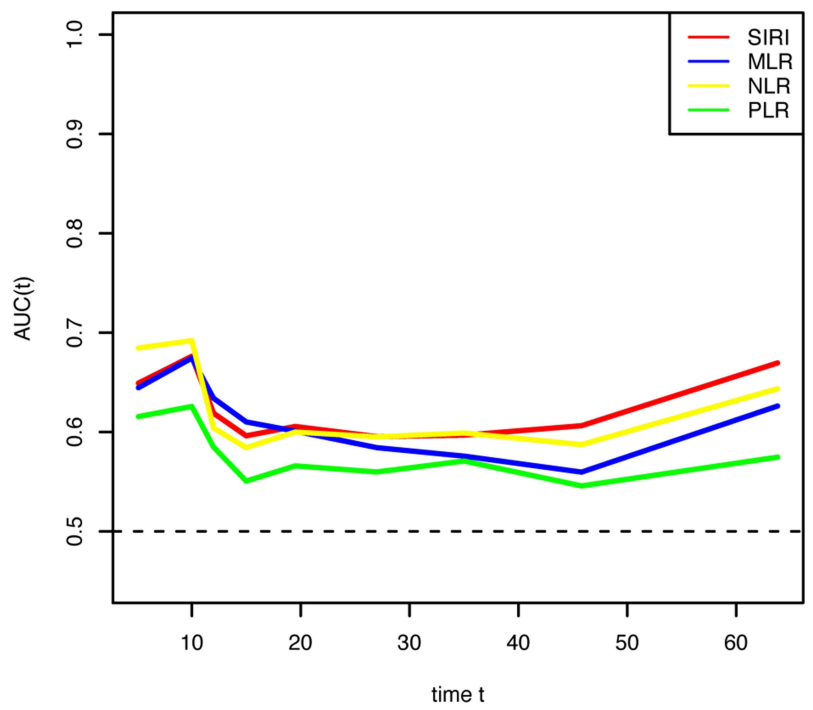

Figure 2 Time-dependent receiver operating characteristic (ROC) analysis of the prognostic model and TNM staging system (A) and inflammation-based biomarkers (B).

Points
SIRI group
PLR group

Figure 3 Prognostic nomogram for predicting I-, 3-, and 5-year overall survival probability based on the systemic inflammation response index (SIRI) group, the platelet-tolymphocyte ratio (PLR) group, maximal tumor size, $N$ stage, $M$ stage, radical surgery, jaundice, and $\gamma$-glutamyl transpeptidase (GGT) level in patients with cholangiocarcinoma.

cholangiocarcinoma is urgently needed in the clinical setting for physicians to make individualized and precise treatment. Although the TNM staging system for cholangiocarcinoma is still the gold standard, limitations such as poor discrimination among patients with the same TNM stage remain unresolved.
Weinberg et al believed that tumor-associated inflammation plays an important role in carcinogenesis and tumor progression. ${ }^{24}$ Accumulating evidence has shown that systemic inflammation-based biomarkers including MLR, NLR, and PLR have prognostic roles in various types of tumors. More specifically, in cholangiocarcinoma, 
A

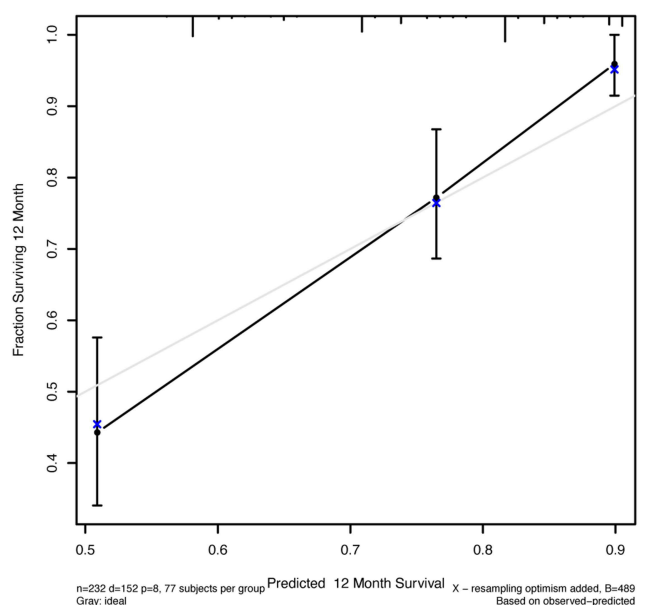

C

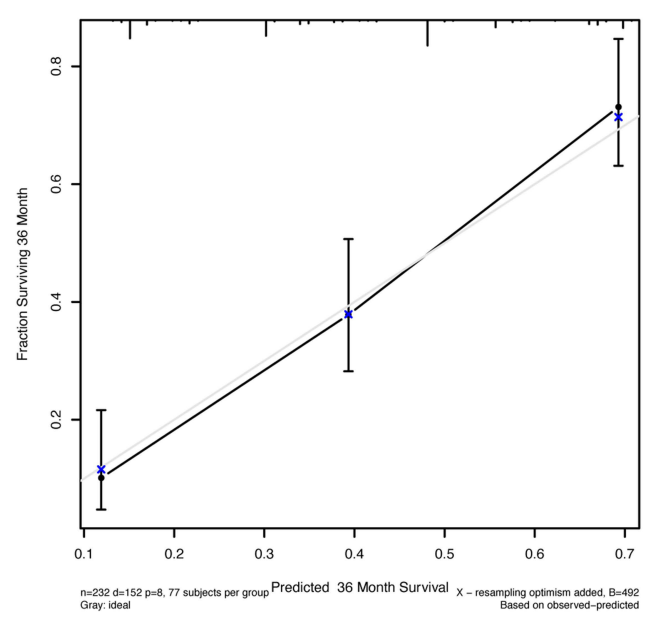

E

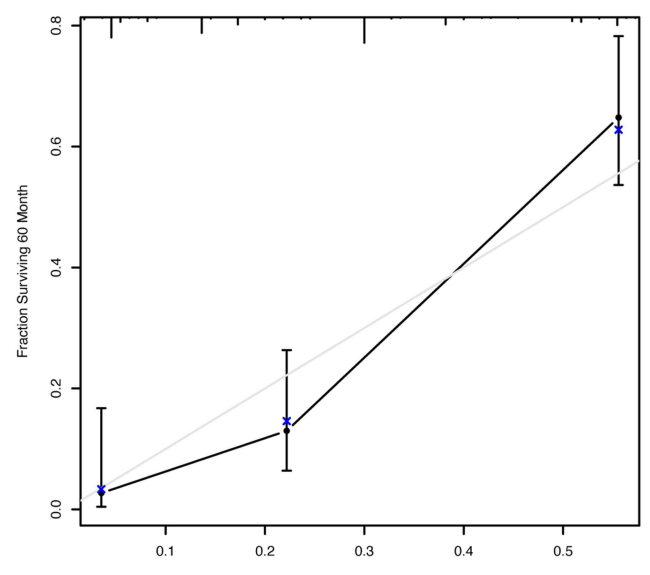

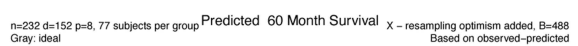

B

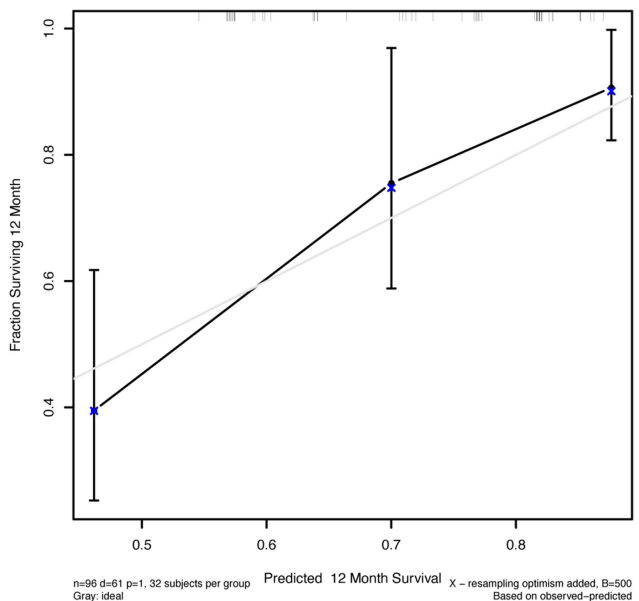

D

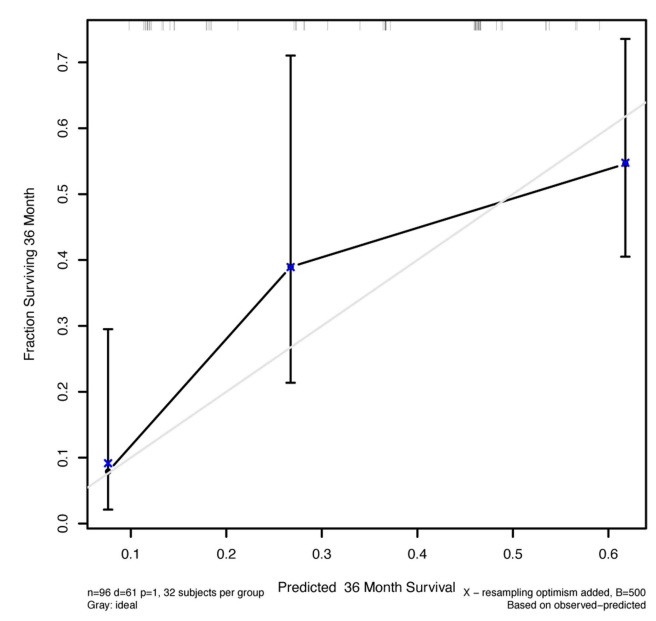

F

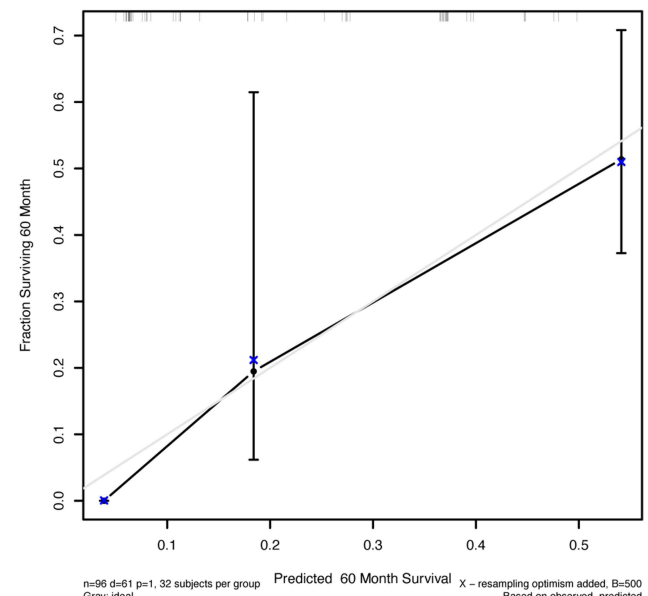

Figure 4 Nomogram calibration plot for predicting overall survival probabilities at I (A and B), 3 (C and D), and 5 (E and F) years in the training and validation cohorts. 
A

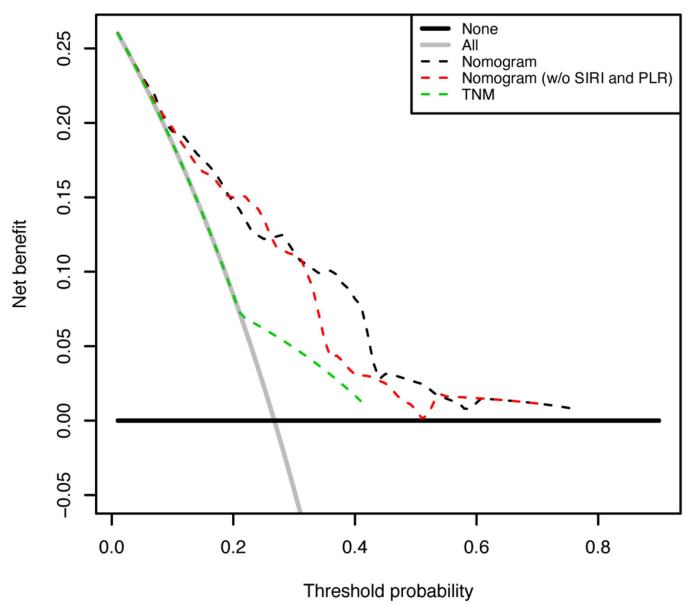

C

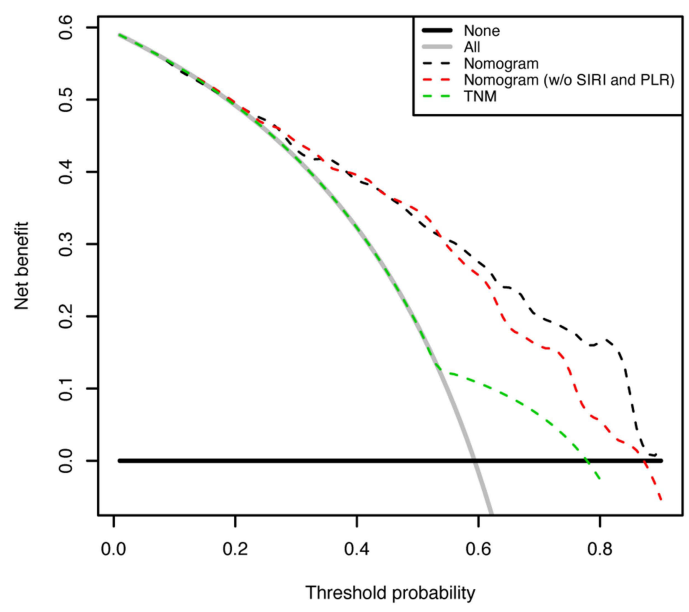

E

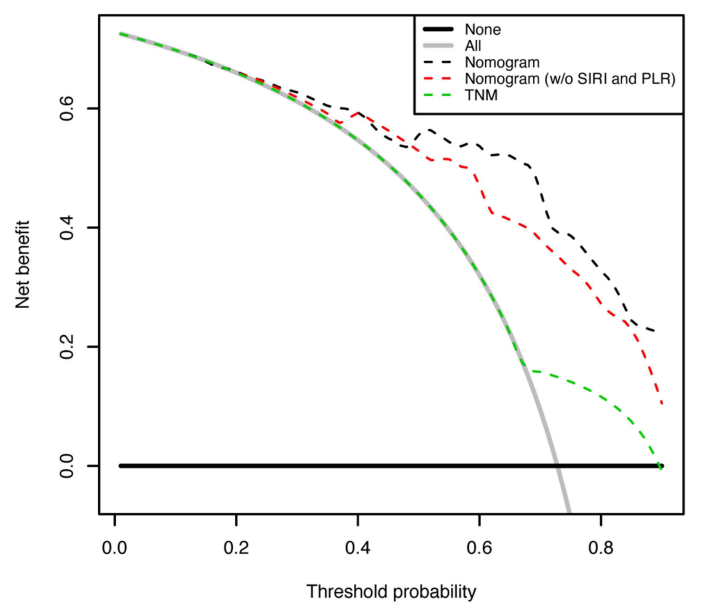

B

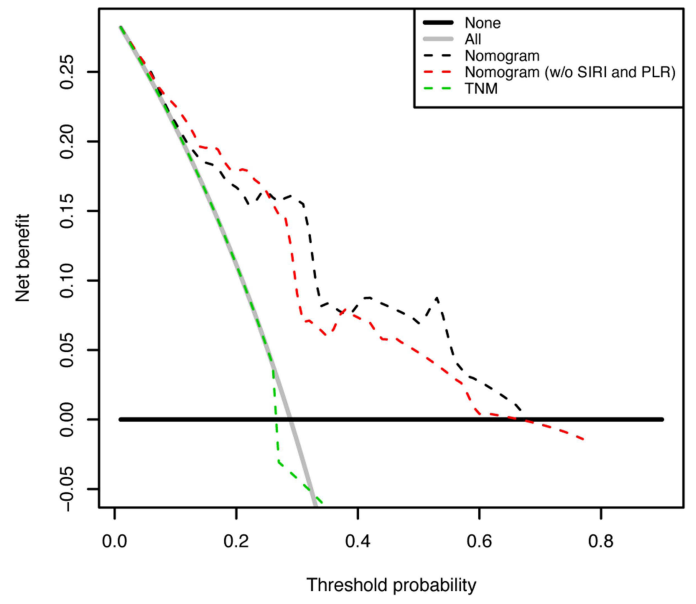

D

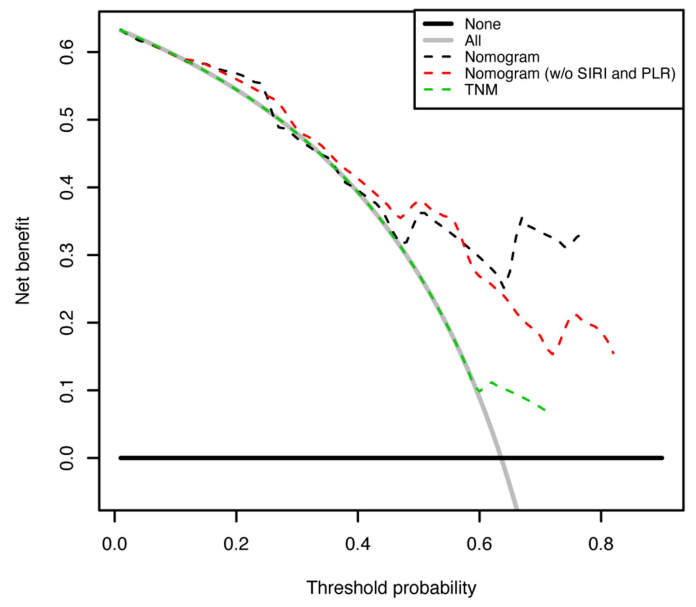

F

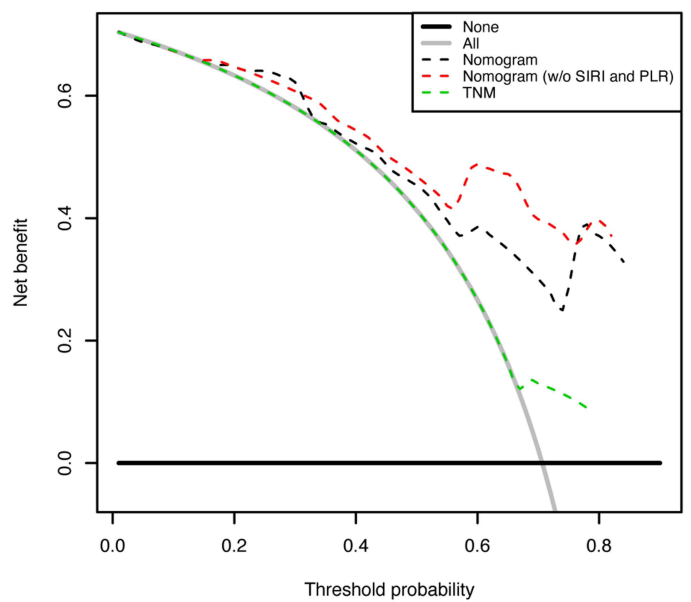

Figure 5 Decision curve analysis of the model and TNM staging system for I- (A and B), 3- (C and D), and 5- (E and F) year survival in the training and validation cohorts. 
A

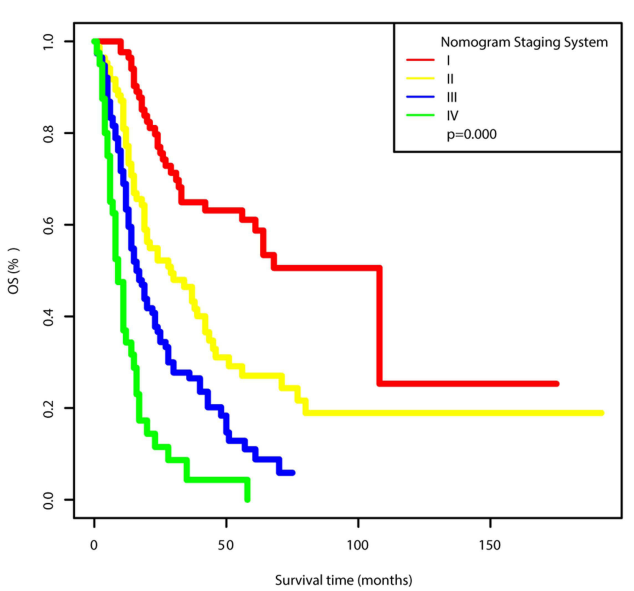

C

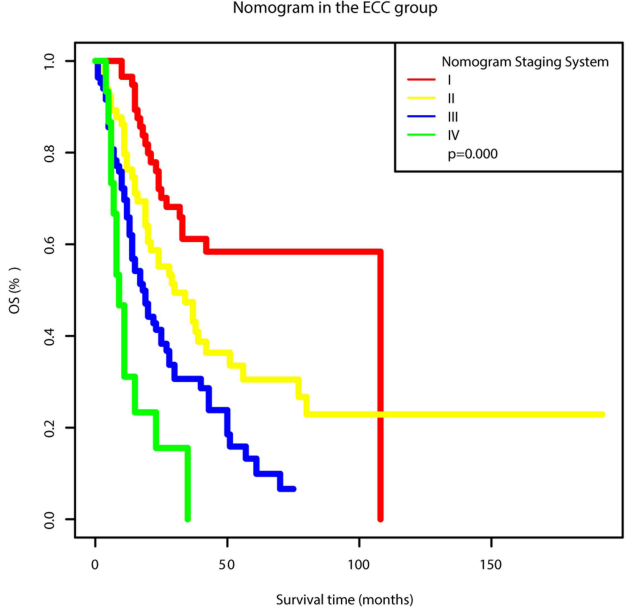

E

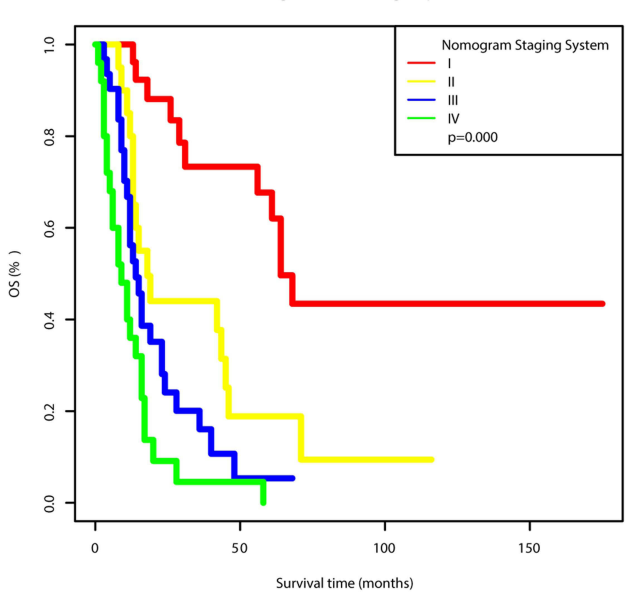

B

TNM

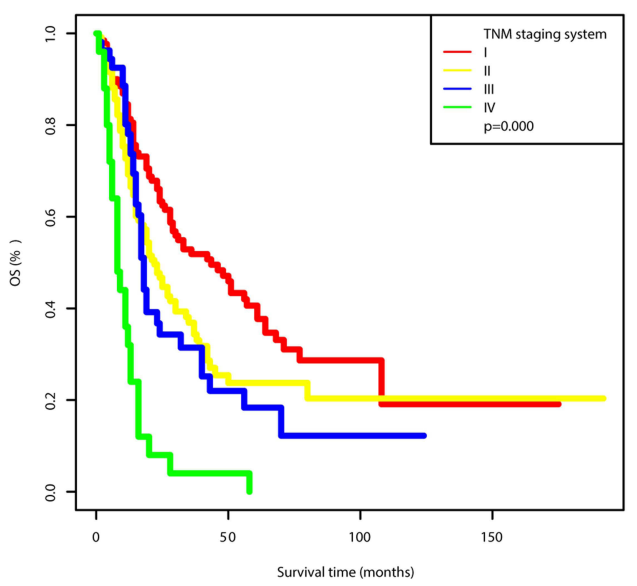

D

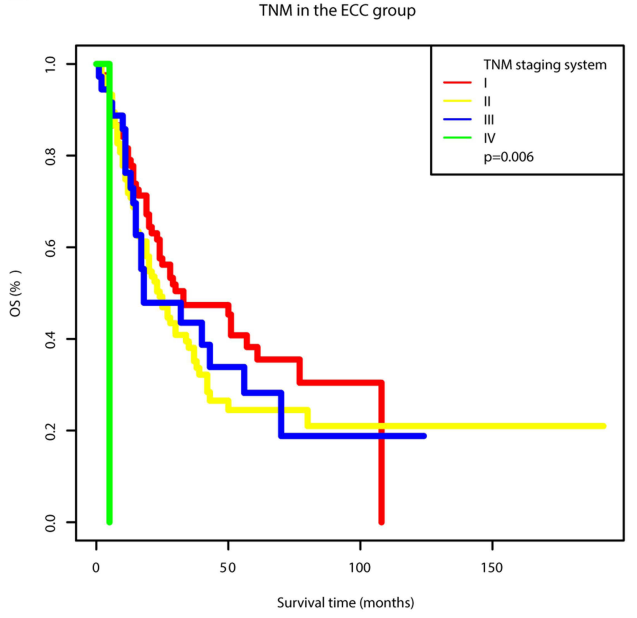

$\mathbf{F}$

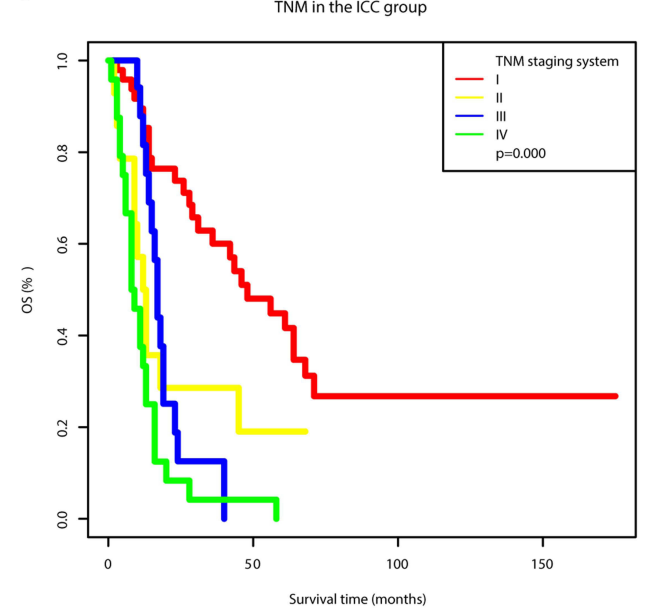

Figure 6 Kaplan-Meier survival curves of the Nomogram and TNM staging system in the whole (A and B), ECC (C and D), and ICC (E and F) cohorts. 
previous studies have found that NLR can predict the survival of patients with advanced biliary tract cancer who receive palliative chemotherapy. ${ }^{10}$ In contrast, the PLR is an independent predictor of OS in patients with recurrent malignant obstructive jaundice. ${ }^{11}$

In the present study, SIRI, an inflammation-based biomarker, was an independent prognostic factor in patients with cholangiocarcinoma. A SIRI value above 0.68 indicated poorer prognosis compared to a SIRI value $\leq 0.68$, and was also correlated with other poor clinicopathological features such as older age, elevated serum LDH, and increased mortality rate. Previous research focusing on other types of solid tumors, such as gastric, esophageal, and nasopharyngeal cancer, had similar results. ${ }^{13-15}$ However, the optimal cutoff value of SIRI differed among studies, which was 1.2 in esophageal cancer and 1.8 in pancreatic cancer. $^{12,14}$ In the present study, we employed 0.68 as the cutoff value to stratify our patients and validate its utility in the validation cohort; however, whether this cutoff applies to all cholangiocarcinoma patients' needs further external inter-institutional validation. Meanwhile, time-based ROC analysis showed that SIRI had better prognostic ability in most time ranges as compared to other inflammation-based biomarkers, including MLR, NLR, and PLR. Multivariate Cox regression analysis showed that only SIRI and PLR were independent prognostic factors among all inflammation-based biomarkers. Based on these findings, we established an innovative prediction model integrating the SIRI, PLR, and other factors, which demonstrated excellent predictive ability in both the training and validation cohorts. In addition, compared to the TNM staging system, the model established in the study showed a much better predictive accuracy with much higher AUCs in 1, 3, and 5 years as well as $\mathrm{C}$-index. Moreover, compared to the model that did not integrate the SIRI and PLR, our model had higher AUCs for the full-time range. Our prediction model also showed better clinical usefulness, as shown by DCA. Furthermore, the Kaplan-Meier curves of the four-group classification based on our model had better discrimination than the TNM staging system in both the whole cohort and the ECC or ICC cohorts. Therefore, SIRI showed potential as a marker for predicting the prognosis of patients with cholangiocarcinoma. Moreover, the model based on the SIRI and other factors had an outstanding predictive value for patients with cholangiocarcinoma after surgical resection. Both the SIRI and prediction model based on it might be applied in the clinical setting to develop more accurate and personalized therapeutic regimens. To our knowledge, this is the first study to construct a nomogram integrating inflammation-based biomarkers, including SIRI and other clinicopathological factors, to predict the prognosis of patients with cholangiocarcinoma, which showed a better predictive ability and clinical utility than those of the model that did not integrate inflammation-based indices.

The specific mechanism by which a high SIRI predicts poor outcome in patients with cholangiocarcinoma is still unclear; however, accumulating evidence has indicated that circulating lymphocytes play an important role in antitumor defense by secreting several types of cytokines such as tumor necrosis factor-alpha (TNF- $\alpha$ ) and interferongamma (IFN- $\gamma)$ to induce cancer cell apoptosis. ${ }^{25,26}$ Thus, a decrease in the number and quality of lymphocytes might result in weakening of the immune system and defense of the tumor. Moreover, the peripheral counting of monocytes is associated with the level of tumorassociated macrophages, which contributes to tumor progression and suppresses the antitumor immune response. $^{27,28}$ Likewise, circulating neutrophils can facilitate tumor angiogenesis by producing vascular endothelial growth factor. $^{29}$ In addition, by secreting reactive oxygen species and nitric oxide, neutrophils can prevent T lymphocyte activation. ${ }^{30}$ Therefore, peripheral neutrophils may be involved in providing a favorable tumor microenvironment to promote tumor growth, invasion, and metastasis. ${ }^{31}$ Taken together, the SIRI combines all three peripheral immune cell counts into a single index to deal with complex interactions. Decreasing lymphocytes and increasing monocytes or neutrophils will cause an elevated SIRI, leading to a worse prognosis for cancer patients. Finally, because of its convenience, noninvasive nature, low price, and reproducibility, the SIRI can be routinely applied during the follow-up period. Both the preoperative value of SIRI and its dynamic changes may serve as biomarkers for the evaluation of the efficacy of adjuvant chemoradiotherapy, immune therapy, or targeted therapy, and for the surveillance of possible recurrence.

The present study has the following limitations. First, this research only studied the inflammation-based biomarkers before surgical resection; however, the dynamic changes of the SIRI and other factors during follow-up may also correlate with patient outcomes, which requires further investigation. Second, this was a single-center retrospective study, which may have led to potential bias. Although the results were validated in a randomly divided 
validation cohort, further external validation is needed before our prognostic model can be widely used. Third, the inclusion of different types of cholangiocarcinoma could amplify the heterogeneity of patients. Although rigorous subgroups analyses have been conducted to shown that the reliability of the results was not obviously compromised by such heterogeneity, researches are needed to confirm the results further in ICC, perihilar cholangiocarcinoma and distal cholangiocarcinoma, respectively. Besides, although we randomly divided the overall population into a training cohort and a validation cohort, some differences in baseline characteristics between the two cohorts may cause potential bias.

\section{Conclusion}

In conclusion, SIRI was an independent prognostic factor in patients with cholangiocarcinoma. This prognostic model integrating SIRI and other clinicopathological factors showed good predictive accuracy in predicting patients' survival probability and was better than the TNM staging system. It may become a reliable tool for doctors to conduct personalized assessments for patients with cholangiocarcinoma.

\section{Abbreviations}

SIRI, systemic inflammation response index; ROC, receiver operating characteristic; TMN, tumor node metastasis; AJCC, American Joint Committee on Cancer; MLR, monocyte-tolymphocyte ratio; NLR, neutrophil-to-lymphocyte ratio; PLR, platelet-to-lymphocyte ratio; PUMCH, Peking Union Medical College Hospital; OS, overall survival; CA 19-9, carbohydrate antigen 19-9; CEA, carcinoembryonic antigen; C-index, Harrell's concordance index; DCA, decision curve analysis; LDH, lactate dehydrogenase; ECC, extrahepatic cholangiocarcinoma; ICC, intrahepatic cholangiocarcinoma; GGT, glutamyl transpeptidase (GGT); ALP, alkaline phosphatase; Alb, albumin; HR, hazard ratio; CI, confidence interval; AUC, area under the receiver operating characteristic curve; TNF- $\alpha$, tumor necrosis factor-alpha; IFN- $\gamma$, interferon-gamma.

\section{Ethics Approval and Informed Consent}

This study was conducted in accordance with the ethical standards of the Declaration of Helsinki and has been approved by the Institutional Review Board of Peking Union Medical College Hospital (Number: S-K1110). Written informed consent was obtained from all patients.

\section{Acknowledgments}

The authors would like to thank Dr. H. Nikki March and Dr. Vikas Narang from Editage, for editing the English text of a draft of this manuscript.

\section{Funding}

This work was supported by grants from the National Natural Science Foundation of China (Grant Number: 81972698), Chinese Academy of Medical Sciences (CAMS) Innovation Fund for Medical Sciences (CIFMS) (Grant Number: 2017-I2M-4-002) and Chen Xiao-Ping Foundation for the Development of Science and Technology of Hubei Province (Grant Number: CXPJJH11900001-2019215).

\section{Disclosure}

The authors have no conflicts of interest to declare.

\section{References}

1. Tyson GL, El-Serag HB. Risk factors for cholangiocarcinoma. Hepatology. 2011;54:173-184. doi:10.1002/hep.24351

2. Rizvi S, Gores GJ. Pathogenesis, diagnosis, and management of cholangiocarcinoma. Gastroenterology. 2013;145:1215-1229.

3. Khan SA, Emadossadaty S, Ladep NG, et al. Rising trends in cholangiocarcinoma: is the ICD classification system misleading us? J Hepatol. 2012;56:848-854. doi:10.1016/j.jhep.2011.11.015

4. Raoof M, Singh G. Rising trends in intrahepatic cholangiocarcinoma incidence and mortality: getting at the root cause. Hepatobiliary Surg Nutr. 2019;8:301-303. doi:10.21037/hbsn.2019.01.15

5. Jarnagin WR, Fong Y, DeMatteo RP, et al. Staging, resectability, and outcome in 225 patients with hilar cholangiocarcinoma. Ann Surg. 2001;234:507. doi:10.1097/00000658-200110000-00010

6. de Jong MC, Marques H, Clary BM, et al. The impact of portal vein resection on outcomes for hilar cholangiocarcinoma: a multi-institutional analysis of 305 cases. Cancer. 2012;118:4737-4747. doi:10.1002/cncr.27492

7. Rizvi S, Khan SA, Hallemeier CL, Kelley RK, Gores GJ. Cholangiocarcinoma - evolving concepts and therapeutic strategies. Nat Rev Clin Oncol. 2018;15:95-111. doi:10.1038/nrclinonc.2017.157

8. McLean L, Patel T. Racial and ethnic variations in the epidemiology of intrahepatic cholangiocarcinoma in the United States. Liver Int. 2006;26:1047-1053.

9. Diakos CI, Charles KA, McMillan DC, Clarke SJ. Cancer-related inflammation and treatment effectiveness. Lancet Oncol. 2014;15: e493-503. doi:10.1016/S470-2045(14)70263-3

10. Ha H, Nam AR, Bang JH, et al. Soluble programmed death-ligand 1 (sPDL1) and neutrophil-to-lymphocyte ratio (NLR) predicts survival in advanced biliary tract cancer patients treated with palliative chemotherapy. Oncotarget. 2016;7:76604-76612. doi:10.18632/oncotarget.2810

11. Jin H, Pang Q, Liu H, et al. Prognostic value of inflammation-based markers in patients with recurrent malignant obstructive jaundice treated by reimplantation of biliary metal stents: a retrospective observational study. Medicine. 2017;96:e5895. doi:10.1097/ MD.0000000000005895

12. Qi Q, Zhuang L, Shen Y, et al. A novel systemic inflammation response index (SIRI) for predicting the survival of patients with pancreatic cancer after chemotherapy. Cancer. 2016;122:2158-2167. doi:10.1002/cncr.30057 
13. Li S, Lan X, Gao H, et al. Systemic Inflammation Response Index (SIRI), cancer stem cells and survival of localised gastric adenocarcinoma after curative resection. $J$ Cancer Res Clin Oncol. 2017;143:2455-2468. doi:10.1007/s00432-017-2506-3

14. Geng Y, Zhu D, Wu C, et al. A novel systemic inflammation response index (SIRI) for predicting postoperative survival of patients with esophageal squamous cell carcinoma. Int Immunopharmacol. 2018;65:503-510. doi:10.1016/j.intimp.2018.10.002

15. Chen Y, Jiang W, Xi D, et al. Development and validation of nomogram based on SIRI for predicting the clinical outcome in patients with nasopharyngeal carcinomas. J Investig Med. 2019;67:691-698. doi:10.1136/jim-2018-000801

16. World Medical Association. Declaration of Helsinki: ethical principles for medical research involving human subjects. J Am Coll Dent. 2014;81:14-18.

17. Edge SB, Compton CC. The American Joint Committee on Cancer: the 7th edition of the AJCC cancer staging manual and the future of TNM. Ann Surg Oncol. 2010;17:1471-1474.

18. Camp RL, Dolled-Filhart M, Rimm DL. X-tile: a new bio-informatics tool for biomarker assessment and outcome-based cut-point optimization. Clin Cancer Res. 2004;10:7252-7259. doi:10.1158/ 078-0432.CCR-04-713

19. Vickers AJ, Elkin EB. Decision curve analysis: a novel method for evaluating prediction models. Med Decis Making. 2006;26:565-574 doi:10.1177/0272989X06295361

20. Saha SK, Zhu AX, Fuchs CS, Brooks GA. Forty-Year Trends in Cholangiocarcinoma Incidence in the U.S.: intrahepatic Disease on the Rise. Oncologist. 2016;21:594. doi:10.1634/theoncologist.20150446

21. Yang JD, Kim B, Sanderson SO, et al. Biliary tract cancers in Olmsted County, Minnesota, 1976-2008. Am J Gastroenterol. 2012;107:1256-1262.
22. Razumilava N, Gores GJ. Cholangiocarcinoma. Lancet. 2014;383:2168-2179. doi:10.1016/S0140-6736(13)61903-0

23. Antwi SO, Patel T. Increasing mortality of intrahepatic cholangiocarcinoma in the US: are gender-specific risk factors important? Hepatobiliary Surg Nutr. 2019;8:635. doi:10.21037/hbsn.2019.10.10

24. Hanahan D, Weinberg RA. Hallmarks of cancer: the next generation. Cell. 2011;144:646-674. doi:10.1016/j.cell.2011.02.013

25. Ferrone C, Dranoff G. Dual roles for immunity in gastrointestinal cancers. J Clin Oncol. 2010;28:4045-4051. doi:10.1200/ JCO.2010.27.9992

26. Rosenberg SA. Progress in human tumour immunology and immunotherapy. Nature. 2001;411:380-384. doi:10.1038/35077246

27. Franklin RA, Liao W, Sarkar A, et al. The cellular and molecular origin of tumor-associated macrophages. Science. 2014;344:921-925. doi:10.1126/science. 1252510

28. Shibutani M, Maeda K, Nagahara H, et al. The peripheral monocyte count is associated with the density of tumor-associated macrophages in the tumor microenvironment of colorectal cancer: a retrospective study. BMC Cancer. 2017;17:404. doi:10.1186/s12885-017-3395-1

29. Kusumanto YH, Dam WA, Hospers GA, Meijer C, Mulder NH. Platelets and granulocytes, in particular the neutrophils, form important compartments for circulating vascular endothelial growth factor. Angiogenesis. 2003;6:283-287. doi:10.1023/B:AGEN.0000029 415.62384.ba

30. Müller I, Munder M, Kropf P, Hänsch GM. Polymorphonuclear neutrophils and $\mathrm{T}$ lymphocytes: strange bedfellows or brothers in arms? Trends Immunol. 2009;30:522. doi:10.1016/j.it.2009.07.007

31. Liu S, Li N, Yu X, et al. Expression of intercellular adhesion molecule 1 by hepatocellular carcinoma stem cells and circulating tumor cells. Gastroenterology. 2013;144:1031-41.e10.
Cancer Management and Research

\section{Publish your work in this journal}

Cancer Management and Research is an international, peer-reviewed open access journal focusing on cancer research and the optimal use of preventative and integrated treatment interventions to achieve improved outcomes, enhanced survival and quality of life for the cancer patient.
The manuscript management system is completely online and includes a very quick and fair peer-review system, which is all easy to use. Visit http://www.dovepress.com/testimonials.php to read real quotes from published authors. 